\title{
Kaposi sarcoma presenting as severe haemoptysis
}

\section{Case report}

\author{
S M A El-Gadi, J Banks, K Yoganathan
}

\section{Case report}

In spring 1995, a 36 year old HIV positive African man with CD4 count of $10 \times 10^{6} / 1$ presented at Singleton Hospital with severe life threatening haemoptysis. Careful examination of the skin and oral cavity showed no evidence of Kaposi sarcoma (KS).

On admission his investigations revealed a platelet count of $30 \times 10^{9} / 1$ and a drop of $5 \mathrm{~g} / \mathrm{dl}$ of haemoglobin to $6.8 \mathrm{~g} / \mathrm{dl}$ compared with a haemoglobin of $11.6 \mathrm{~g} / \mathrm{dl} 3$ weeks previously. Chest radiograph showed persistent shadowing in the right mid zone and computed tomography (CT) revealed bilateral perihilar pulmonary infiltrates with small bilateral pleural effusions. Extensive bleeding KS lesions throughout the bronchial tree were found on bronchoscopy (fig). He was transfused and commenced on tranexamic acid. His clotting times were slightly abnormal, which were corrected with fresh frozen plasma and intravenous vitamin $K$. Although he received only one dose of parenteral vincristine and bleomycin, the haemoptysis gradually settled over 3 weeks. The patient's condition' deteriorated and he developed hepatomegaly with obstructive jaundice. Blood culture was negative but culture for mycobacterium avium complex (MAC) was not performed. The ultrasound appearance was suggestive of liver abscess and despite high doses of parenteral antibiotics he died 4 weeks after admission. Postmortem examination was declined.

\section{Discussion}

Pulmonary $\mathrm{KS}$ is common in African HIV positive patients, occurring in $46 \%$ of cases, ${ }^{1}$ perhaps because patients tend to present late and as a result of the more common existence of Kaposi's sarcoma associated herpesvirus (KSHV) in African patients. ${ }^{2}$ While haemoptyses are reported in one third of cases, they are usually small. Simultaneous cutaneous KS may be absent in 5-23\% of patients with symptomatic pulmonary KS. Chronic cough and progressive dyspnoea are the most common presenting symptoms of pulmonary $\mathrm{KS}$, occurring in $67-100 \%$ and in $83-100 \%$ of cases respectively, lower thoracic pain in $17-47 \%$, and a high fever in $30-53 \%$, but in most cases it is related to concomitant infection. The mean CD4 count in patients with pulmonary $\mathrm{KS}$ is $96 \times 10^{6} / 1$, but pulmonary KS has been reported with more than $400 \times$ $10^{6} / 1 .{ }^{3}$ Physical examination may reveal crackles, wheeze, or stridor in patients with $\mathrm{KS}$ of the upper respiratory tract. Chest radiograph may be normal in up to $20 \%$ of cases, or may show parenchymal densities that follow septal lines. Single or multiple nodular opacities may also be found and both abnormalities are associated in $70 \%$ of cases, and when in combination are highly suggestive of pulmonary KS. Less common radiological abnormalities include segmental, lobar, alveolar, interstitial, and atelectasis. The frequency of intrathoracic lymphadenopathy and pleural effusion varies widely. The latter when present is usually large, bilateral, and when associated with parenchymal lesions is highly suggestive of KS. In a series evaluating CT scanning in 61 patients with intrathoracic KS, the association of two or more pulmonary nodules, peribronchovascular thickening, pleural effusions, and tumour masses on CT were found in $75 \%$ of patients. ${ }^{4}$

The characteristic endoscopic appearance is sufficient for a presumptive diagnosis to be made once opportunistic infection and pulmonary tuberculosis, particularly in African patients, are excluded. Endobronchial lesions may not be visible at bronchoscopy, and parenchymal lesions may be patchy and could be missed at transbronchial biopsy. Open lung biopsy may therefore be useful in the absence of endobronchial lesions. Occasionally, bronchopulmonary $\mathrm{KS}$ is difficult to distinguish from Pneumocystis carinii pneumonia (PCP). While it is desirable to carry out bronchoalveolar lavage to exclude associated pulmonary infection, particularly before chemotherapy, this may not be feasible in every patient.

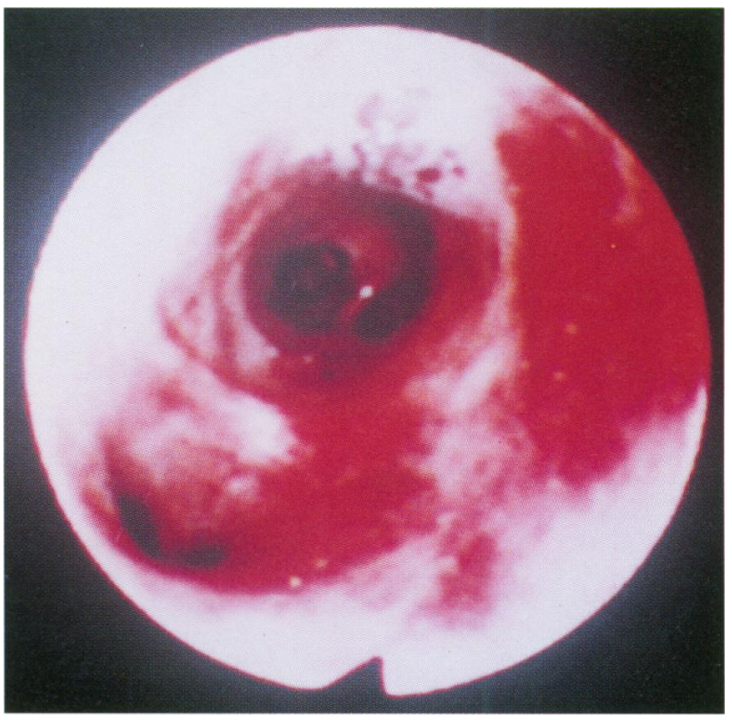

Bronchoscopy showing endobronchial lesions of Kaposi sarcoma. 
Alveolar lavage was physically impossible in this case because of the degree of haemoptysis. Intrathoracic $\mathrm{KS}$ does not take up gallium and patients with nodular and/or interstitial abnormalities on chest radiograph that do not fix gallium are likely to have KS. Ground glass areas of attenuation on CT was virtually diagnostic of P.CP in $92 \%$ of cases in one study. ${ }^{5}$

This is the first documented case of pulmonary KS presenting with severe life threatening haemoptysis, and illustrates that even in the absence of mucocutaneous lesions a high index of suspicion is required in patients with AIDS who present with blood stained sputum and abnormal chest radiograph.

1 Pozniak AL, Latif AS, Neill P, Houston S, Cheu K, Robertson V. Pulmonary Kaposi sarcoma in Africa. Thorax 1992;47:730-3.

2 Chang $\mathrm{Y}$, Ziegler J, Wabinga $\mathrm{H}$, Katangote-Mbidde $\mathrm{E}$ Boshoff C, Schulz T, et al. Kaposi's sarcoma-associated herpesvirus and Kaposi's sarcoma in Africa. Ugand Kaposi's sarcoma study group. Arch Intern Med 1996; 156:202-4.

3 Cadranel J, Kammoun S, Chevret S, Parrot A, Denis $M$ Winter C, et al. Results of chemotherapy in 30 AIDS patients with symptomatic pulmonary Kaposi's sarcoma. Thorax 1994;49:958-60.

4 Cadranel J, Mayaud C. Intrathoracic Kaposi's sarcoma in patients with AIDS. Thorax 1995;50:407-14.

5 Hartman TE, Primack SL, Muller NL, Staples CA Diagnosis of thoracic complications in AIDS: accuracy of CT. Am $\mathcal{F}$ Roentgenol 1994;162:547-53. 